\title{
Network analysis of atherosclerotic genes elucidates druggable targets
}

\author{
Sheuli Kangsa Banik, Somorita Baishya, Anupam Das Talukdar and Manabendra Dutta Choudhury*
}

\begin{abstract}
Background: Atherosclerosis is one of the major causes of cardiovascular disease. It is characterized by the accumulation of atherosclerotic plaque in arteries under the influence of inflammatory responses, proliferation of smooth muscle cell, accumulation of modified low density lipoprotein. The pathophysiology of atherosclerosis involves the interplay of a number of genes and metabolic pathways. In traditional translation method, only a limited number of genes and pathways can be studied at once. However, the new paradigm of network medicine can be explored to study the interaction of a large array of genes and their functional partners and their connections with the concerned disease pathogenesis. Thus, in our study we employed a branch of network medicine, gene network analysis as a tool to identify the most crucial genes and the miRNAs that regulate these genes at the post transcriptional level responsible for pathogenesis of atherosclerosis.

Result: From NCBI database 988 atherosclerotic genes were retrieved. The protein-protein interaction using STRING database resulted in 22,693 PPI interactions among 872 nodes (genes) at different confidence score. The cluster analysis of the 872 genes using MCODE, a plug-in of Cytoscape software revealed a total of 18 clusters, the topological parameter and gene ontology analysis facilitated in the selection of four influential genes viz., AGT, LPL, ITGB2, IRS1 from cluster 3. Further, the miRNAs (miR-26, miR-27, and miR-29 families) targeting these genes were obtained by employing MIENTURNET webtool.

Conclusion: Gene network analysis assisted in filtering out the 4 probable influential genes and 3 miRNA families in the pathogenesis of atherosclerosis. These genes, miRNAs can be targeted to restrict the occurrence of atherosclerosis. Given the importance of atherosclerosis, any approach in the understanding the genes involved in its pathogenesis can substantially enhance the health care system.
\end{abstract}

Keywords: Atherosclerosis, Network medicine, Gene network analysis, Cytoscape, STRING, AGT, LPL, ITGB2, IRS1, miRNA, MIENTURNET

\section{Background}

Atherosclerosis is a multifactorial inflammatory disease of the arteries. It is the major cause of most cardiovascular diseases like myocardial infarction, peripheral artery disease and stroke [86]. According to World Heart Federation, cardiovascular disease is the leading cause of mortality worldwide, causing 17.9 million deaths per

*Correspondence: drmdc@bioinfoaus.ac.in

Department of Life Science and Bioinformatics, Assam University, Silchar, India year (www.world-heart-federation.org/world-heart-day/ world-heart-day-2019/cvds, accessed on 11/02/2021). Atherosclerosis is characterized by the accumulation of modified low density lipoprotein (LDL) in the arteries that are exposed to turbulent flow of blood, thereafter triggers a series of inflammatory responses. Initially monocytes are recruited to bind to the endothelium and then they migrate into the sub endothelial cells and convert to macrophages that engulf the modified LDL and form foam cells. This induces proliferation of smooth muscle cell, accumulation of extra cellular matrix protein 
and results in the formation of atherosclerotic plaque that hinders the regular flow of blood [13, 27, 67].

Studies have shown that the pathophysiology of atherosclerosis involves the interplay of a number of genes and metabolic pathways, hence they are greatly targeted to overcome the atherosclerotic complications [16]. The cholesterol transport system is an important component in the regulation of atherosclerosis and the genes associated with itlike APOA1, ABCA1, ABCG1, LCAT, APOB, CETP, LPL, PPAR $\gamma$, LXR, SR-A, FoxO1, PCSK9,etc. were investigated immensely to gain scientific insights in order to establish them as a target $[48,86]$. Also, the genes that are involved with the inflammatory responses like VCAM1, MCP1, M-CSF, IL-1 $\beta$, TNF $\alpha$ and $\beta$, IL-6, M-CSF, MCP-1, IL-18 and CD-40L, etc. were thoroughly studied to elucidate their role in atherosclerosis[21]. Thus, from an array of genes, prioritization of gene/s that is/are key regulator of atherosclerosis becomes difficult from the translational aspect.

In this regard, Network Medicine (NM), a very recent and promising discipline that aids in the identification of disease genes, pathways and the probable targets for drugs can be utilized. This NM approach takes under consideration the system biology and network science to provide a deeper insight in the perturbation of a disease in a holistic way. NM encompasses the integration of large biological data sets (genomics, proteomics, etc.) and creates desired network type (protein-protein interaction, metabolomics, etc.) to elucidate the connections among the biological components and thereafter revealing the influential pathophenotype [12, 20, 30, 58]. Here under the framework of NM, gene network analysis was conducted to predict the most influential gene/s in the pathogenesis of atherosclerosis. This in-silico approach facilitates to reconstruct the interactions between genes, proteins, signaling pathways associated with metabolic disorders by using low and high throughput experimental and computational data having statistical significance [72]. In this study, a protein-protein interaction network of the concern disease involving gene/protein and their associated partners was constructed. Further based on the cluster formation and gene ontology analysis, the key regulatory gene can be identified $[3,10]$. Gene network analysis has been successfully used by Sarajlic et al. to identify the 'driver genes' that are involved in cardiovascular diseases [65]. In coronary artery disease the Combined Network Topological Features was explored to identify the crucial gene involved with the disease [88]. Gene network analysis has also employed in predicting novel genes/therapeutic drug targets of human genes infected by COVID 19, meniere's disease, chronic obstructive pulmonary disease It has also helped in the identification of driver epigenetic factor, tumor suppressor genes and the genes responsible for glioblastomaTherefore, gene network analysis facilitates to design drug for various infections and also for varioius metabolic diseases [28, 55, 90, $9240,19,39]$. It also aids in identification of sub-networks of diseased genes that are associated with varied disorders by interactome based network analysis [56]. An investigation was conducted on the switch genes of different cancer types caused by BRAF ${ }^{\mathrm{V} 600 \mathrm{E}}$ mutant in order to understand the mechanisms of the varied response exhibited by a drug, vemurafenib [26]. Thus, it can be seen that gene network analysis has a wide range of implication in the biological domain.

Gene network analysis assists in the identification of influencial genes and these genes are inturn regulated by miRNA. Nowadays, clinical researches exploit miRNA as a therapeutic target to treat various diseases like coronary heart disease, hepatitis $\mathrm{C}$, liver cancer, diabetes, etc. miRNAs are the short non-coding RNA molecules formed from a long primary RNA transcript and are expressed in the peripheral blood, cerebrospinal fluid, saliva, urine, and other biological samples. A single miRNA has the potential to target a large number of mRNA and regulate the expression of gene. The treatment regime includes either blocking the expression of a specific miRNA or compensating the expression loss of miRNA [31,[76]. Various studies have shown that when miRNA was targeted the pathophysiology of CVD could be obsoleted, like when the expression of miR-378 was enhanced cardiac congestion could be prevented and when the expression of miR-133 was inhibited the occurrence of hyperthrophy was reduced [18], the downregulation of miR-1 and miR-26 and the upregulation of miR-21 and miR-328 along with the alteration of other miRNAs have helped in the reduction of Atrial fibrillation [82].

\section{Method \\ Retrieval of genes}

The genes associated with atherosclerosis were retrieved from National Centre for Biotechnology Information (NCBI) database (https://www.ncbi.nlm.nih.gov/gene; Accessed on 22/12/2020) using the query words "atherosclerosis, Homo sapiens".

\section{Protein-protein interaction}

The protein-protein interaction (PPI) data were generated by using the freely available database STRING (Search Tool for the Retrieval of Interacting Genes/ Proteins) version 11 (https://string-db.org/). STRING encompasses both physical and functional aspects of proteins and takes into account evidences from experimental works, databases mining, pathway databases, coexpressed and neighborhood gene analysis, fusion and 
co-occurrence of protein association/distribution analysis. The interactions among the proteins are depicted by confidence scores that range between 0 and 1 . The highest confidence score lies between 0.9 and 1 , high confidence score between 0.7 and 0.9 , medium confidence score between 0.4 and 0.7 and low confidence score is less than 0.4. In the network, each gene is designated as node and the interactions between the nodes are designated as edge $[4,69,70]$

\section{Construction of gene interaction network}

The visualization model of biological network is generated by using Cytoscape (3.4.0) Software. It is afreely available software with various other plug-in apps viz., MCODE for clustering analysis, BiNGO/ClueGo for functional enrichment analysis, AgilentLiteratureSearch for literature mining, etc. The network obtained from PPI analysis was imported to Cytoscape in a network specific format such as SIF (Simple Interaction File). Cytoscape merges, visualize and analyse the STRING networks using various algorithm for network layout like springembedded layout, hierarchical layout, and circular layout $[64,70]$.

\section{Gene clustering analysis}

MCODE (Molecular COmplexDEtection), a Cytoscape plug-in is a density-based clustering algorithm that identifies the clusters of densely connected nodes from the network by generating a score and rank. It operates in three levels: Firstly it weighs all nodes using $k$-core value. Secondly, centering the highest weighted node the cluster is predicted. Thirdly, it undergoes post-processing depending on core value. The resulting complexes are then scored, ranked accordingly and graphical modules are obtained $[9,64]$, and hence enhances the reliability of MCODE. Clustering analysis by MCODE has aided in the identification of cruicial genes in the pathophysiology of atherosclerosis and also in other diseases [57, 73].

\section{Topological parameter analysis}

The topological parameters analysis depicts the arrangement of interacting nodes within a network. Cytoscape plugin Network Analyzer takes into account topological parameters like node degree betweeness centrality, and average shortest pathlength. The number of edges extending out of a node determines its degree. Significant biological interactions are considered to be associated with high node degree. Average Shortest Pathlength is the measure of the least distance between two nodes. Betweenness centrality of a node is the summation of all the shortest pathlength from onenode to another connected either directed or indirectly $[6,84]$.

\section{Gene ontology and pathway interrelation analysis}

ClueGo in association with CluePedia were used for gene ontology and pathway enrichment analysis. This cytoscape plug-in facilitates the prediction of biological pathway conferred by genes which can be visualized as networks of functionally grouped terms. It uses the precompiled GO terms to calculate the enrichment analysis for GO terms, GO term fusion, mid- $p$-values, Bonferroni step-down method and $\mathrm{p}$ value less than equals to 0.5 were used, keeping predefined kappa threshold value [15]

\section{miRNA target prediction}

The prediction of miRNA that targets the obtained influential genes was facilitated by the web tool MIENTURNET (MicroRNA ENrichment TURned NETwork). This tool takes under consideration both the computational and experimental evidences from TargetScan and miRTarBase websites respectively to predict the interaction of miRNA on target gene. The input for MIENTURNET could be either gene list or miRNA list and a network is obtained that is statistically significant. The analysis of the network was done by computing the topological parameters, and performing functional enrichment analysis [41].

\section{Result}

Construction of gene networks of atherosclerotic genes The query for "Atherosclerosis, Homo sapiens" in the NCBI database revealed a total of 988 genes that were involved in atherosclerosis (Additional file 1: Table S1). The input of all 988 genes in STRING revealed that 96 genes were not found in the database. Thus, networks with 892 genes were constructed at different confidence scores. At medium confidence score 22,693 protein-protein interactions were found among 872 nodes (Additional file 2: Table S2c) whereas at high confidence score 8314 protein-protein interactions were found among 777 nodes (Additional file 2: Table S2b), and at highest confidence score 5248 protein-protein interactions were obtained among 675 nodes (Additional file 2: Table S2a).

\section{Cluster analysis}

Using MCODE, the densely associated nodes in the protein-protein interaction network were obtained as clusters. Total 18 clusters were obtained and were ranked based on their scores (density to node ratio). Out of 872 nodes, only 465 nodes having 6607 edges (interaction) were associated with cluster formation whereas others remained unclustered. The 18 clusters along with the scores and name of associated nodes are 
Table 1 List of clusters as obtained by MCODE analysis

\begin{tabular}{|c|c|c|c|c|}
\hline Cluster & $\begin{array}{l}\text { Score } \\
\text { (Density*\#Nodes) }\end{array}$ & Nodes & Edges & Node IDs \\
\hline 1 & 69.631 & 158 & 5466 & $\begin{array}{l}\text { VEGFA, VEGFC, AKT1, PDGFRB, STAT3, NLRP3, NOS3, TLR7, CASP3, STAT1, CD28, CD86, JUN, CD40LG, } \\
\text { CD40, PTEN, APOB, ESR1, RHOA, APOE, APOA1, HIF1A, TP53, MAPK1, KDR, MAPK3, PTGS2, ACE, AGTR2, } \\
\text { FASLG, MMP2, ACKR3, C5, FLT1, BDKRB1, C3, GNB3, GPR55, ANXA5, MTNR1B, GPER1, P2RY12, SUCNR1, } \\
\text { IL6R, GRM8, SAA1, IFNG, HCAR2, FPR1, FPR2, CNR1, CASR, APLN, ANXA1, NGF, CCL28, APLNR, CCR2, NPY, } \\
\text { CXCR5, CCR5, CXCR6, PPBP, PECAM1, CXCR4, PF4, TLR4, CXCL5, TLR6, CXCL16, CCL5, CXCL1, CXCL12, } \\
\text { CXCL8, TNFRSF1A, TNFSF11, MPO, IL18, ELANE, NOTCH1, IL15, ADAM10, LGALS1, SERPIND1, TIMP1, } \\
\text { PROC, IGFBP5, FGF23, APOA2, ICAM1, STC2, KITLG, F5, MIA3, VCAM1, QSOX1, APOA5, APOL1, TNC, } \\
\text { MMP9, PCSK9, AHSG, SERPINA1, CP, CD44, MMP3, C4A, SPP1, TLR2, IGFBP1, CSF1, TLR1, MFGE8, IGFBP3, } \\
\text { CST3, GAS6, PLAU, MMP1, ALB, FN1, IL6, CDH1, ITGB1, BDNF, TNF, HMOX1, IL17A, VWF, CCL2, ADIPOQ, } \\
\text { IL10, IL1B, CRP, IDO1, LEP, PPARG, CD68, FOXP3, SELL, INS, CD34, PTPRC, PROM1, ENG, CCL11, TNFSF13B, } \\
\text { EDN1, HMGB1, SELE, RELA, ELN, SRC, JAK2, HGF, TGFB1, IGF1, SERPINE1, PLG }\end{array}$ \\
\hline 2 & 12.615 & 66 & 410 & $\begin{array}{l}\text { KLRK1, BMP2, IGF2, IL9, SOCS3, SIRT1, HBEGF, CAT, JAG1, SELP, SNAI1, GPR29, LCN2, TBX21, MIF, NOS2, } \\
\text { MMP10, MMP7, MTOR, CYBB, PLAUR, MMP14, AR, CTNNB1, EGF, IL7R, AGTR1, SOD2, TNFSF10, ADAM17, } \\
\text { GPT, CCL17, F3, FCGR2A, IL5, SYK, CDC42, RETN, CCL22, CX3CL1, CD163, ANGPT1, CD69, ANGPT2, PGF, } \\
\text { KIT, F2, NFKB1, CXCR2, CX3CR1, CXCR3, SOCS1, HAVCR2, IGF1R, IL33, CDH5, CXCL13, CDKN2A, HSPA4, } \\
\text { BGLAP, LGALS3, NOD2, GJA1, IL1A, CLEC7A, IL1RN }\end{array}$ \\
\hline 3 & 10.217 & 84 & 424 & $\begin{array}{l}\text { CTSB, IL22, IL23R, IL23A, BRCA1, OSM, MMP8, CDKN1B, SOD1, TREM1, HSPA5, KLF4, CAMP, IRS1, TERT, } \\
\text { NOX1, NOX4, NCF1, JAK1, DCN, ITGB2, IL2RA, CD4, FOXO1, WNT5A, VLDLR, ILK, AVP, FOXO3, IKBKB, LYN, } \\
\text { SELPLG, PLTP, ITGB3, ABCB1, TIMP3, LCAT, B2M, PLAT, TXN, ALOX5, TNFRSF11B, TNFRSF9, NR3C1, AGT, } \\
\text { ITGA4, AHR, LPL, GGT2, GCG, VDR, P2RY2, APOA4, SAA2, VIMP, GGT1, CETP, AGER, APOC3, PON1, UTS2, } \\
\text { HPR, MSR1, KISS1R, APOC1, GHSR, EDNRA, APOC2, UTS2R, PLA2G7, UTS2B, EDN3, MCAM, KISS1, APOBR, } \\
\text { CYSLTR1, EDNRB, LTB4R, LIPC, NFE2L2, APOH, TNFAIP3, COL18A1, TNFSF4 }\end{array}$ \\
\hline 4 & 5.3 & 41 & 106 & $\begin{array}{l}\text { LEPR, DICER1, CDKN3, PGLYRP1, BMP4, HSPD1, HSPB1, ITGA2B, PTX3, DKK1, ADIPOR1, ITGA2, UCP1, PTH, } \\
\text { CHIT1, LIPG, CALCA, HPSE, NEU1, EZH2, CYP19A1, BGN, CD14, SREBF2, NPC1L1, MTTP, DNMT1, EPHA2, } \\
\text { SIRT6, ITGAV, CTSK, VIM, SCARB1, ACP5, PPARA, ESR2, STK11, VCL, ABCG8, ABCG5, SREBF1 }\end{array}$ \\
\hline 5 & 5.282 & 40 & 103 & $\begin{array}{l}\text { CTSL, SCD, GC, NAMPT, UCP2, LTA4H, FADS1, RBP4, FABP4, KLF2, MDK, FGF21, TNFAIP6, ANGPTL3, } \\
\text { ADIPOR2, ADM, ANGPTL4, CTSS, HLA-DRB1, MAPK7, HSPG2, GATA2, GHRL, HP, LPA, OLR1, PLCG1, ADRB2, } \\
\text { F2R, LDLR, ABCA1, RARRES2, HMGCR, IRS2, NR1H3, ABCG1, ACTA2, NOTCH2, S100A9, NRG1 }\end{array}$ \\
\hline 6 & 4.8 & 6 & 12 & LTC4S, TBXAS1, ALOX15, ALOX12, PLA2G6, PLA2G2A \\
\hline 7 & 3.333 & 4 & 5 & HABP2, F12, HGFAC, PROZ \\
\hline 8 & 3.333 & 4 & 5 & DHX38, THOC5, HNRNPC, DHX15 \\
\hline 9 & 3.2 & 6 & 8 & ACSL1, FABP3, CPT1A, NR4A1, UBE2I, NCOA2 \\
\hline 10 & 3 & 3 & 3 & MSTN, MEF2C, PPARGC1A \\
\hline 11 & 3 & 7 & 9 & NUMB, CLTCL1, AAK1, PSMA6, PSMD6, SCARB2, LTBR \\
\hline 12 & 3 & 3 & 3 & ADAMTS3, ADAMTS1, COMP \\
\hline 13 & 3 & 3 & 3 & NAT2, GSTM1, GSTO1 \\
\hline 14 & 3 & 3 & 3 & DDAH2, DDAH1, ARG2 \\
\hline 15 & 3 & 3 & 3 & PAFAH1B2, PLA2G10, PAFAH1B3 \\
\hline 16 & 3 & 3 & 3 & RNF111, RNF213, HERC6 \\
\hline 17 & 2.846 & 27 & 37 & $\begin{array}{l}\text { ADAMTS4, ADAMTS5, TLN1, ADAMTS13, CAP1, GSTP1, MAN2B1, THBS2, AKR1B1, RIPK2, CHI3L1, PIK3CB, } \\
\text { ORMDL3, CD36, CDK5, PLIN2, XDH, ELAVL1, CTSD, YWHAZ, ADAM8, PRDX1, TNFSF12, ARSB, TRAF2, } \\
\text { TNFRSF25, CTSC }\end{array}$ \\
\hline 18 & 2.667 & 4 & 4 & GLS2, TSHB, GCLC, TXNRD2 \\
\hline
\end{tabular}

listed in Table 1. The individual clusters are depicted in Additional file 8: Figure S1.

\section{Network analysis}

The Cytoscape tool, network analyzer facilitated in the analysis of all the 18 clusters. The topological parameters like node degree distribution, betweenness centrality, and average shortest pathlength were analysed for all the clusters. The topological parameters of all the clustered nodes are listed in Additional file 3: Table S3.

\section{Biological function and pathway analysis}

ClueGo in association with CluePedia, a cytoscape plugin was used for functional enrichment analysis. Using the default network specificity of the plug-in, the enrichment of all the 18 clusters was done. Out of which Cluster 1 , $2,3,4,5,6$ were found to be functionally enriched. For cluster 1, 310 Gene Ontology (GO) terms were obtained; for cluster 2, $26 \mathrm{GO}$ terms were obtained; for cluster 3, 27 GO terms were obtained; for cluster 4, 3 GO terms were obtained; for cluster 5, 8 GO terms were obtained, 
and for cluster 6,1 GO term was obtained. The list of all the GO terms of 6 clusters alongside the biological processes and genes involved are given in Additional file 4: Table S4. A flow chart of the network analysis has been depicted in Fig. 1.

\section{MiRNA-target interaction}

The genes AGT, LPL, IRS1, and ITGB2 were uploaded as official gene symbol in MIENTURNET web tool. The statistical analysis was performed by miRNA-Target enrichment using both TargetScan and miRTarBase database to over-represent the miRNA-Target interaction. TargetScan generated miRNA family hsa-miR-29a-3p/ hsa-miR-29b-3p/hsa-miR-29c-3p and hsa-miR-27a-3p/ hsa-miR-27b-3p. These miRNAs interacted with IRS1 and LPL by binding with seeds (conserved regions) AGC ACCA and UCACAGU respectively. Whereas, miRTarBase generated one miRNA, hsa-miR-26b-5p and this interacted with IGBT2 and AGT (Fig. 2, Table 2). The topological parameters of the nodes are provided in Additional file 5. The disease ontology of the miRNAs revealed a total of 124 and 95 GO terms using TargetScan and miTarBase database respectively (Additional file 6). Of which $30 \mathrm{GO}$ terms obtained from TargetScan and 16 GO terms obtained from miTarBase database are associated with atherosclerosis either directly or indirectly.

\section{Discussion}

Cardiovascular Disease (CVD) is the leading cause of death in the world and atherosclerosis being the major cause of CVD poses a serious health concern [68]. The pathophysiology of atherosclerosis involves the interplay of a number of inflammatory genes, lipid genes, also genes involved with diabetes and results in the manifestation of atherosclerotic plague $[45,63]$. In the present study, in silico gene network analysis was used as a tool to prioritize the most influential gene/s among all the atherosclerotic genes that have key regulatory role in the pathogenesis of atherosclerosis. The reliability of in silico gene network analysis to predict influencial genes was elucidated by Baishya et al. in their recent publication [11]. For this study, 988 atherosclerotic genes were obtained from NCBI database; these genes were used to construct PPI using STRING. Subsequently, cluster analysis, topological parameters analysis and functional enrichment analysis helped in identifying 4 genes viz., AGT (Gene ID: 183), LPL (Gene ID: 4023), ITGB2 (Gene ID: 3689), and IRS1(Gene ID: 3667) from cluster 3 that may be considered to be most influential in the
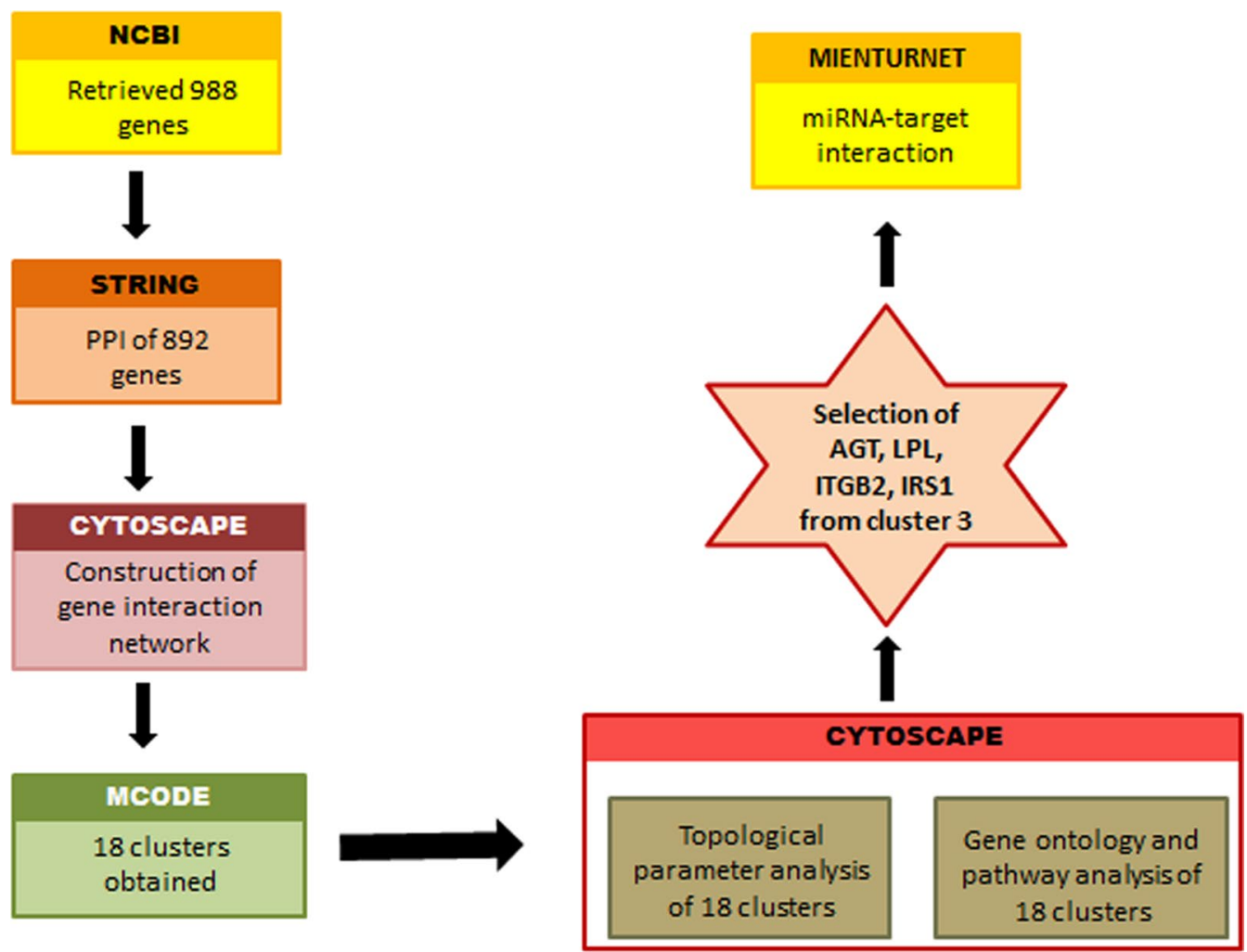

Fig. 1 A flowchart of the network analysis 


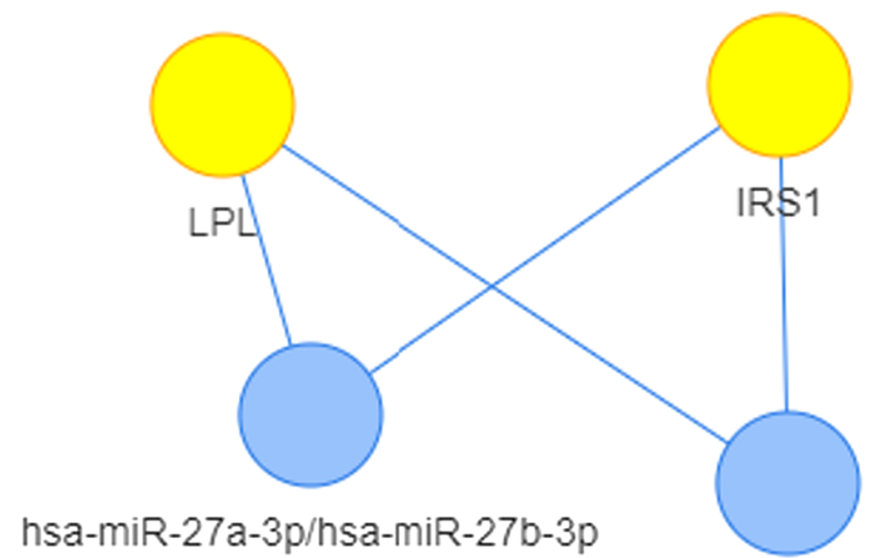

hsa-miR-29a-3p/hsa-miR-29b-3p/hsa-miR-29c-3p

\section{a)Computational prediction of miRNA-Target obtained by using TargetScan database}

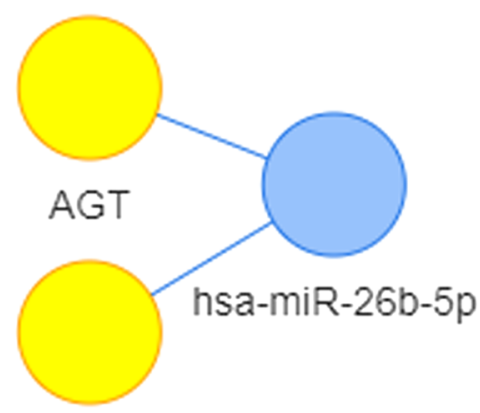

ITGB2

b)Experimentally validated miRNA-Target obtained by using miTarBase database

Fig. 2 miRNA-Target interaction using MIENTURNET

Table 2 miRNA-Target interaction

\begin{tabular}{|c|c|c|c|c|c|c|}
\hline Seed & miRNA family & $p$ value & Odd ratio & $\begin{array}{l}\text { Number of } \\
\text { interactions }\end{array}$ & Target Gene 1 & Target Gene 2 \\
\hline \multicolumn{7}{|c|}{ Mienturnet Enrichment results using TargetScan } \\
\hline AGCACCA & $\begin{array}{l}\text { hsa-miR-29a-3p / hsa-miR-29b-3p } \\
\text { / hsa-miR-29c-3p }\end{array}$ & 0.009408396 & 0.097031526 & 2 & IRS1 & $L P L$ \\
\hline UCACAGU & hsa-miR-27a-3p/hsa-miR-27b-3p & 0.011872998 & 0.108997469 & 2 & IRS1 & LPL \\
\hline microRNA & $p$ value & Odd ratio & Number of interactions & Target Gene 1 & Target Gene 2 & \\
\hline \multicolumn{7}{|c|}{ Mienturnet Enrichment results using miRTarBase } \\
\hline hsa-miR-26b-5p & 0.078148643 & 0.248805098 & 2 & ITGB2 & AGT & \\
\hline
\end{tabular}

pathogenesis of atherosclerosis. Based on the MCODE score and functional enrichment analysis, cluster 3 was selected. Further on the basis of degree of nodes, betweenness centrality, and average shortest path length the genes having $\geq 100$ degree were selected. The lists of selected genes are tabulated in Table 3. A pie diagram depicting the GO groups of cluster 3 is represented in Fig. 3 
Table 3 List of selected genes

\begin{tabular}{|c|c|c|c|c|c|}
\hline Name & MCODE Cluster & MCODE-Score & $\begin{array}{l}\text { Avg. shortest } \\
\text { pathlength }\end{array}$ & Betweeness centrality & Degree \\
\hline AGT & ["Cluster 3"] & 27.08641975 & 1.91618829 & 0.00803355 & 161 \\
\hline LPL & ["Cluster 3"] & 27 & 2.00918485 & 0.0040675 & 113 \\
\hline ITGB2 & ["Cluster 3"] & 25.69500675 & 2.06429392 & 0.00339199 & 104 \\
\hline IRS1 & ["Cluster 3"] & 24.83599419 & 1.9793341 & 0.00162085 & 100 \\
\hline
\end{tabular}

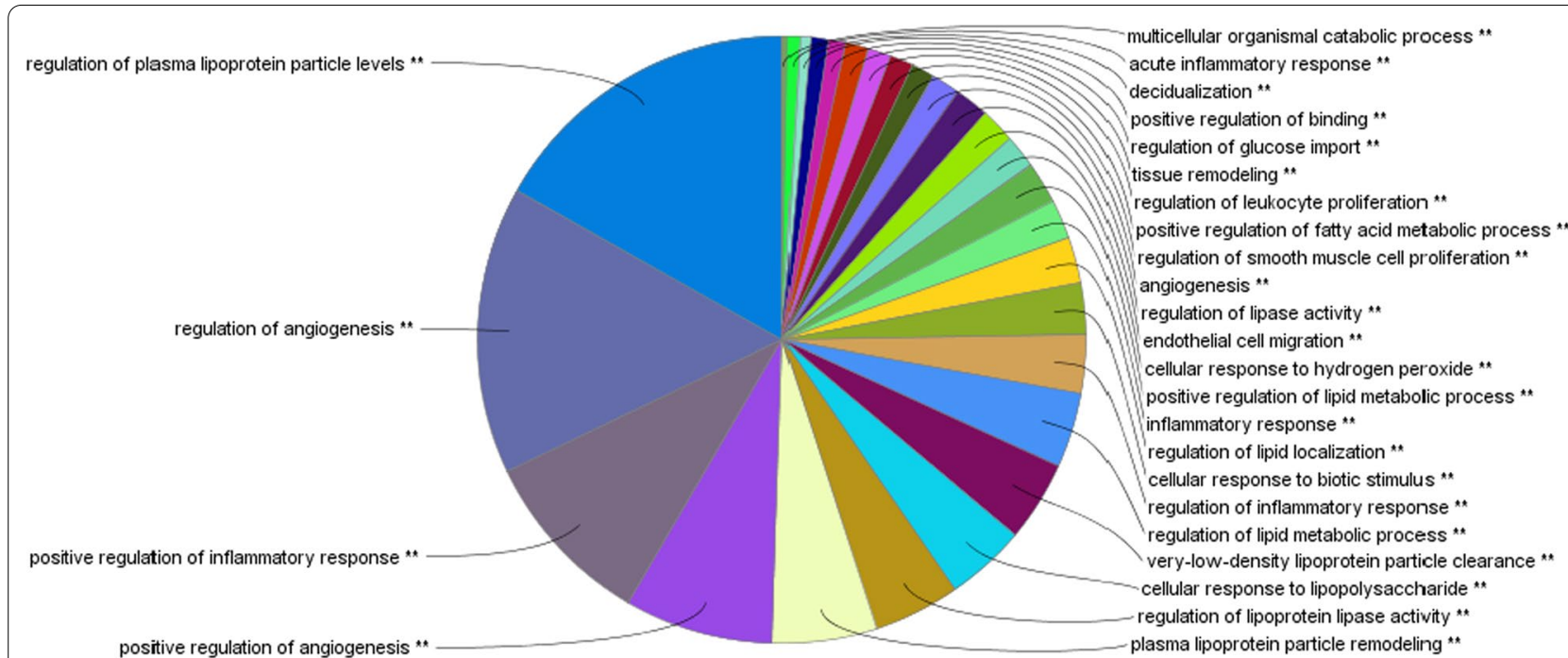

Fig. 3 Pie chart depicting the $27 \mathrm{GO}$ groups of cluster 3 generated by ClueGo. The area covered by each group represents the number of GO terms within each group. The most significant term of the group is labelled

AGT encodes for angiotensinogen and is found to be associated with $111 \mathrm{GO}$ terms viz., angiogenesis, regulation of lipase activity, vasoconstriction, regulation of blood pressure, regulation of ion homeostasis, renal system process, etc. involved in different GO groups. The list consisting of GO terms is tabulated in Additional file 7: Table S7a. Primarily, angiotensinogen is produced by liver and is the precursor of other angiotensins [85]. Angiotensin is a component of Renin-Angiotensin-Aldosterone (RAAS) system or Renin-Angiotensin system (RAS) and has a key implication on cardiovascular, renal, neural, endothelial system $[66,85]$. When the sodium concentration in kidney is low, renin is secreted. It then acts at the $10 \mathrm{~N}$-terminal amino acid of angiotensinogen (AGT) to produce Angiotensin I (AngI), further angiotensin converting enzyme (ACE) catalyses the conversion of AngI to Angiotensin II (AngII) [29]. AngII and AngI are also catalyzed by ACE2 to produce Angiotensin 1-7. In systemic arterioles, AngII binds to Angiotensin II type 1 receptor (AT1) and Angiotensin II type 2 receptor (AT2) and acts as vasoconstrictor by enhancing fluid intake and increasing blood pressure. Whereas Ang1-7 act on Mas receptor and results in the synthesis of nitric oxide and prostaglandin and thereby causes vasodialation [29, 36, 85]. AngII stimulates the release of aldosterone and antidiuretic hormone which activates sodium reabsorption, potassium excretion, and water reabsorption in kidney respectively to maintain the blood volume and pressure [29]. AngII is however responsible to elicit atherosclerosis by recruiting monocytes, promoting macrophages lipid peroxidation and increasing oxidative stress. Hong $\mathrm{Lu}$ et al. found that when drugs that inhibit Renin were administered to LDL receptor deficient mice the progression of atherosclerosis was prevented by inhibiting the production of AngI [42]. Daugherty et al. have found that when AngII was infused in APOE deficient mice, the progression of atherosclerotic lesion was increased by influencing sheer stress in the artery, activation of monocytes and monocyte chemoattractant Protein-1 [22]. AngII causes oxidation of Low Density Lipoprotein (LDL) which in turn is taken up by the receptors of macrophages and leads to the formation of atherosclerotic 
lesion. Studies have shown that the inhibition of binding of AngII to ATI receptor can prevent the development of atherosclerosis [23, 37].

Also,when renin acts at the $10 \mathrm{~N}$-terminal amino acid of AGT, only $2 \%$ of it produces AngI but the remaining 98\% of AGT known as des(AngI)AGT has independent role to perform in angiogenesis. In a study it was seen that synthesis of low concentration of AGT reduces body weight gain and liver steatosis which is contrast to AngII dependent function but the underlying mechanism is not clear. Thus, AngII independent AGT functions can be proved beneficial to hypertensive obesed individual. Also, when AGT was inhibited, the blood pressure and the development of atherosclerotic lesion was decreased, this is similar to the finding where when AngII was inhibited the blood pressure and development of atherosclerotic lesion was reduced. However, owing to the role in embryonic development of RAS system, the complete deletion of major RAS system can lead to low neonatal survival rate $[22,43]$.

LPL encodes for the enzyme lipoprotein lipase. It has been found that it is associated with 41 GO terms viz., inflammatory response, plasma lipoprotein particle remodeling, cholesterol homeostasis, regulation of macrophage derived foam cell differentiation, etc. involved in different GO groups. All are listed in the Additional file 7: Table S7b. LPL acts as a rate limiting enzyme by hydrolyzing the triglyceride present on Very Low Density Lipoprotein (VLDL) and chylomicron to free fatty acid and glycerol. The fatty acid so formed are taken up by tissues and acts as an energy source for cardiac muscle [59]. Dysfunction of LPL system can lead to the manifestation of atherosclerosis, chylomicronemia, dyslipidemia, obesity [50]. The LPL system has 3 important components: LPL, Apolipoprotein CII, glycosylphosphatidylinositolanchored high density lipoprotein-binding protein 1 (GPIHBP1). Any anomality in these components can lead to hypertriglyceridemia [54]. Experiment on homozygous knockout mice has led to the development of hypertriglyceridemia, an increased level of VLDL and reduced adipose tissue store because triglycerides could not be hydrolysed and thus fatty acids could not be stored in adipose tissue [79]. Invitro study has revealed that LPL has differential role in expression of inflammatory genes that play a key role in atherosclerosis, it was seen that LPL had the ability to suppress TNFo-induced gene expression by inhibiting NF-B activation and induced IFN $\gamma$ gene expression by activating STAT1 pathway [38]. In macrophage of LPL knockout mice, the plasma LPL and lipoprotein remained unchanged, cholesterol ester formation and intracellular triglyceride level was reduced and thus interfere with the development of atherosclerosis $[8,71]$. There is also evidence that with the increase in the level of LPL, monocyte derived macrophage activation is triggered and the activator of LPL, APOC2 is also increased. These macrophages get laden with lipid to form foam cells which a vital step in the progress of atherosclerosis [51].

ITGB2 encodes for beta 2 intrigrin protein, it was found that it is associated with $19 \mathrm{GO}$ terms viz., angiogenesis, inflammatory response, nitric oxide metabolic process, etc. involved in different GO groups. (Additional file 7: Table S7c). ITGB2 is transmembrane adhesion and signaling receptor expressed exclusively on leukocytes and extracellular vesicles. It has two sub units: a $\beta$ chain (CD18) and one of the $\alpha$ chains-CD11a (LFA1), CD11b (Mac-1), CD11c (p150, 95), or CD11d. It aids in the adhesion of leukocytes and its migration into the intima [25, 27, 52]. Once in the intima it triggers different key inflammatory molecules and immune mediators like ICAM1, VCAM1 thereby activating endothetial cells and stimulate macrophages to take up modified lipoproteins. Thus, aids in the process of atherosclerosis [25]. A study has shown that in CD18 knockout mice, the manifestation of atherosclerosis was prevented and also increased the capacity of macrophages to take up more modified lipoproteins and apoptotic cells. Thus, exhibiting a direct relationship of ITGB2 with atherosclerosis [25]. Another study on CD11d and APOE knockout mice revealed that CD11d has a proatherogenic role because the absence of CD11d has reduced the formation foam cells, proinflamatory cytokines which are the key steps in atherosclerosis [7]. An investigation by Huaizhu Wu et al. was done to determine the role of CD11c in hypercholesterolemia and atherogenic lesion and it was found that on APOE double deficiency hypercholesteromic mice the expression of CD11c was increased and the number of circulating monocytes were increased compared to wild type APOE double deficiency normal fed mice. And deficiency of CD11c on APOE double deficiency mice reduces the firm attachment of monocytes to VCAM1, P Selectin which are proinflamatory cytokine for the development of atherosclerosis [80].

IRS1 encodes for insulin receptor substrate 1 and is found to be associated with $40 \mathrm{GO}$ terms viz., regulation of lipid metabolic process, regulation of glucose import, regulation of fatty acid metabolic process, etc. involved in different GO groups. All the GO terms are listed in the Additional file 7: Table S7d. IRS1 is a member of Insulin receptor family, which is a cytoplasmic adapter protein. IRS1 is mainly associated with glucose metabolism; in an experiment it was found that ubiquitinization and degradation of IRS1 resulted in the immobilization of GLUT4 transporter in adipocytes and impair its glucose uptake potency [47], 78. A similar result was found in another experiment that involved IRS1 knockout mice lacking 
hepatic IRS2, it was observed that leptin, IGF1 levels was decreased, glucose tolerance ability was impaired and resulted in hyperglycemia [24]. The development of hyperglycemic condition due to the degradation of IRS1 causes nonenzymatic glycosylation of lipids and proteins, and eventually triggers oxidative stress and activates protein kinase $\mathrm{C}$ (PKC). These lead to the modification of LDL and interferes with the LDL-Receptor mediated clearance of LDL, that further triggers the activation of monocyte derived macrophages to generate foam cells and thereby causing atherosclerosis [5]. In an experiment it was found that in IRS1 knockout mice during the hyperinsulinemic-euglycemic clamp condition, the plasma glycerol level and plasma free fatty acid were greatly reduced as compared to IRS2 deficient and wild type mice suggesting that IRS1 has more lipolysis suppressing potential than IRS2 [60]. Also, Sterol regulatory element binding protein 1c (SREBP1c) regulate the process of lipid metabolism by modulating many lipolytic gene expression. It was observed that the expression of the SREBP-1c gene and glucokinase gene were reduced when primary hepatocyte culture was infected with dominant-negative mutant of PI 3-kinase. Thus, IRS1- phosphatidylinositol (PI) 3-kinase pathway plays an important role in insulin metabolism by regulating the expression of SREBP-1c [49].

\section{Predicted atherosclerotic genes as druggable target}

Based on literature mining and database search it was found that drugs like eplerenone, cilazapril, eprosartan are available that targets the renin, ACE, AT1 respectively of RAAS system by blocking their activity [87] (https:// go.drugbank.com/, accessed on 12/02/2021). However to the best of our knowledge, no reports have been found that assert AGT as drug target of any synthesized drug. Since AGT is the only precursor for the derivation of the entire angiotensin family, targeting this could be proven to be effective. LPL is associated with the hydrolysis triglyerides and alteration in the normal physiology can lead to hypertriglyceridemia. Glycyrrhizic Acid a natural compound of licorice plants has been found to reduce the level of LPL expression (Yoke Yin, So Ha, \& Abdul Kadir, 2010). However, no synthesized drug has been found which acts on LPL as a target. ITGB2 that encodes Beta 2 Integrins are exploited as drug target for autoimmune diseases. A drug BMS-587101 blocks CD11a activity and is known to prevent inflammation [14]. Thus, this can be a potential drug target for atherosclerosis which is an inflammation mediated pathogenesis. A natural compound Carainterol A extracted from Caragana intermedia was found to increase the level of IRS1, therapeutic intervention mediated through IRS1 was considered to be a novel approach in treating insulin resistance, as discussed previously insulin sensitivity is associated with hyperglycemic condition, hence IRS1 can also be targeted for treating atherosclerosis [46]. Thus, it was evident from the literature mining and database search that all the 4 genes can be treated as a potential drug target to mitigate the glitches of atherosclerosis.

\section{miRNA as a potential therapeutic}

miRNAs are short, non-coding nucleotides that bind with mRNAs and regulate the expressions of proteins. Thus, altering the expression of miRNA can influence the pathophysiology of any disease. In-silico in vitro and/or in vivo approaches have been successfully employed in establishing miRNA as potential drug target. The initiation of clinical trial of Miravirsen, a miRNA-122 repressor and RG-101 for the treatment of hepatitis $C$ virus have set up the beginning of the upcoming era of miRNA based drugs $[17,31]$. Role of miRNAs in the pathophysiology of cardiovascular disease have been studied extensively and are regarded as potential drug targets $[18,62$, 93].

miRNA-Target prediction revealed miRNA families that target IRS1 and LPL are hsa-miR-27a-3p/hsamiR-27b-3p and hsa-miR-29a-3p/hsa-miR-29b-3p/ hsa-miR-29c-3p, and IRS1 and ITGB2 are targeted by hsa-miR-26b-5p. A number of papers reported that miRNAs are associated with different cardiovascular complications [62, 93]. The reduced level of miR-27a, miR-29a, and miR-26b cause heart failure [93]. Down regulation of miR-26 is associated with atrial fibrillation, thus overexpression reduces the susceptibility of atrial fibrillation [44]. The over-expression of miR-26a/b causes alteration in the cholesterol synthesis [53]. When miRNA expression profiling was conducted in atherosclerotic model, exhibiting hypertension and hyperlipidemia, there was a significant increase in the expression of miR-26b along with other miRNAs [2]. Also, down regulation of miR-26a triggers angiotensin II-induced fibrogenesis in cardiac fibroblasts resulting in the occurrence of hypertensive myocardial fibrosis [90, 92]. The upregulation of miR-27b causes cardiac hypertrophy and its suppression cause inhibition of hypertropic cell growth [77]. miR-27a up-regulation suppresses the differentiation of adipocytes which are the primary sites of cholesterol metabolism [1, 61], up-regulation of miR-27b is reported to be associated with dyslipidemia and atherosclerosis [75]. miR-29a is known to target IRS1 and alter the Akt/GSK-3 pathway and glucose uptake, it also impairs endothelial cells function; miR-29c is suspected to have antiangiogenic effect [91]. miR-29 regulates the genes that are responsible for lipid metabolism, miR-29a up-regulation enhances the chances of insulin resistence [81]. It was observed that the expression of miR-29 was down-regulated in the area 
of fibrotic lessions that elucidates the role of miR-29 in causing cardiac fibrosis [74]. A drug called Miragen that targets miR-29 is already in active phase 1 trial to treat keloid and scar tissue [31]. Apart from it, other drugs that target different miRNAs are also in clinical trials [89]. Thus, it could be understood from the above discussion that the regulatory role of miRNAs are very critical and hence they are nowadays considered as new generation therapeutics to combat various metabolic diseases.

\section{Conclusion}

Owing to the seriousness of atherosclerosis the identification of the key genes that play influential role in causing this condition is the need of the hour. From the vast array of genes that are associated with this condition, AGT, LPL, ITGB2, IRS were identified to play vital role in the pathogenesis of atherosclerosis. AGT is the primary precursor of angiotensins and aid in the regulation of angiogenesis and blood pressure, LPL hydrolyzes triglyceride of VLDL and chylomicron. ITGB2 facilitates the adhesion and migration of leukocytes and generate inflammatory response. IRS1 is primarily associated with glucose metabolism and hyperglycemia. However, these genes are regulated by miRNAs: miR-26, miR-27, and miR-29 families. miRNAs are non-coding nucleotides that are associated with various diseases by regulating the expression of respective genes at the post transcriptional level. Hence, either these genes or the miRNAs interacting with these genes could be regarded as potential targets for therapeutic drug intervention of atherosclerosis. However, translational validation is essential for the final establishment of these genes as possible atherosclerotic drug target.

\footnotetext{
Abbreviations

PPI: Protein-protein interaction; CVD: Cardiovascular disease; GO: Gene ontology; LPL: Lipoprotein lipase; AGT: Angiotensinogen; IRS1: Insulin receptor substrate; ITGB2: Beta 2 integrin; ACE: Angiotensin converting enzyme; AT1: Angiotensin II type 1 receptor; SREBP: Sterol regulatory element-binding transcription factor; PKC: Protein kinase C; RAAS: Renin- angiotensin-aldosterone system; IGF: Insulin-like growth factor; VCAM: Vascular cell adhesion molecule; APOE: Apolipoprotein E; ICAM: Intercellular adhesion molecule; APOC2: Apolipoprotein C2; STAT: Signal transducer and activator of transcription; IFN: Interferon; TNF: Tumor necrotic factor; VLDL: Very low density lipoprotein; Ang: Angiotensin; LDL: Low density lipoprotein; ABCA1: ATP-binding cassette transporter; LCAT: Lecithin-cholesterol acyltransferase; CETP: Cholesteryl ester transfer protein; PPAR: Peroxisome proliferator-activated receptors; LXR: Liver $X$ receptor; SR-A: Scavenger receptor $A$; FoxO1: Forkhead box protein O1; PCSK9: Proproteinconvertasesubtilisin/kexin type 9; MCP: Monocyte chemoattractantprotein; M-CSF: Macrophage colony-stimulating factor; IL: Interleukin; GPIHBP1: Glycosylphosphatidylinositol-anchored high density lipoproteinbinding protein 1.
}

\section{Supplementary Information}

The online version contains supplementary material available at https://doi. org/10.1186/s12920-022-01195-y.
Additional file 1. List of genes involved in atherosclerosis.

Additional file 2. Protein protein interaction at different confidence score.

Additional file 3. Topological parameter of all the clustered nodes obtained by MCODE.

Additional file 4. List of all GO terms of 6 clusters obtained from ClueGo.

Additional file 5. Topological parameter of nodes obtained by MIENTURNET.

Additional file 6. Disease ontology of miRNA obtained by MIENTURNET.

Additional file 7. List of biological processes mediated by 4 genes obtained by ClueGo.

Additional file 8. Clusters generated after MCODE analysis.

Acknowledgements

Authors are grateful to DBT- Bioinformatics Infrastructure Facility (BIF) of Assam University, India for software and infrastructure support, and DelCON's e-Journal Access Facility for e-journal access facility.

\section{Authors' contributions}

SKB and SB carried out the experiment. MDC designed the overall framework of the work. SKB wrote the manuscript under the supervision of ADT and MDC. All authors read and approved the final manuscript.

\section{Funding}

Not applicable.

\section{Availability of data and materials}

The datasets analysed during the current study are available and in the NCBI repository and can be downloaded, (https://www.ncbi.nlm.nih.gov/gene, and accessed on 22/12/2020). The accession numbers are provided in the Additional file 1

\section{Declarations}

Ethics approval and consent to participate

Not applicable.

\section{Consent for publication}

Not applicable.

\section{Competing interests}

The authors declare that they have no competing interests.

Received: 21 April 2021 Accepted: 18 August 2021

Published online: 03 March 2022

\section{References}

1. Aguilar D, Fernandez ML. Hypercholesterolemia induces adipose dysfunction in conditions of obesity and nonobesity. Adv Nutr. 2014;5(5):497-502.

2. Alexandru N, Constantin A, Nemecz M, Comarita IK, Vîlcu A, Procopciuc A, Georgescu A, et al. Hypertension associated with hyperlipidemia induced different microRNA expression profiles in plasma, platelets, and plateletderived microvesicles; effects of endothelial progenitor cell therapy. Front Med. 2019:6:280.

3. Anitha P, Anbarasu A, Ramaiah S. Gene network analysis reveals the association of important functional partners involved in antibiotic resistance: a report on an important pathogenic bacterium Staphylococcus aureus. Gene. 2016;575(2):253-63.

4. Anitha P, Bag S, Anbarasu A, Ramaiah S. Gene and protein network analysis of AmpC $\beta$ lactamase. Cell Biochem Biophys. 2015;71(3):1553-67.

5. Aronson D, Rayfield EJ. How hyperglycemia promotes atherosclerosis: molecular mechanisms. Cardiovasc Diabetol. 2002;1(1):1-10. 
6. Assenov Y, Ramírez F, Schelhorn S-E, Lengauer T, Albrecht M. Computing topological parameters of biological networks. Bioinformatics. 2007:24(2):282-4.

7. Aziz MH, Cui K, Das M, Brown KE, Ardell CL, Febbraio M, Ballantyne CM, et al. The upregulation of integrin aD 22 (CD11d/CD18) on inflammatory macrophages promotes macrophage retention in vascular lesions and development of atherosclerosis. J Immunol. 2017;198(12):4855-67.

8. Babaev VR, Fazio S, Gleaves LA, Carter KJ, Semenkovich CF, Linton MF. Macrophage lipoprotein lipase promotes foam cell formation and atherosclerosis in vivo. J Clin Investig. 1999;103(12):1697-705.

9. Bader GD, Hogue CW. An automated method for finding molecular complexes in large protein interaction networks. BMC Bioinformatics. 2003:4(1):2.

10. Baishya S, Banik SK, Talukdar AD, Anbarasu A, Bhattacharjee A, Choudhury MD. Full title: Identification of potential drug targets against carbapenem resistant Enterobacteriaceae (CRE) strains using in silico gene network analysis. Gene Reports. 2019;14:129-37.

11. Baishya S, Deshamukhya C, Wangkheimayum J, Das BJ, Anbarasu A, Talukdar AD, Choudhury MD, et al. Transcriptional expression of secondary resistance genes $\mathrm{ccdB}$ and repA2 is enhanced in presence of cephalosporin and carabapenem in Escherichia coli. 2020.

12. Barabási A-L, Gulbahce N, Loscalzo J. Network medicine: a network-based approach to human disease. Nat Rev Genet. 2011;12(1):56-68.

13. Barquera S, Pedroza-Tobías A, Medina C, Hernández-Barrera L, BibbinsDomingo K, Lozano R, Moran AE. Global overview of the epidemiology of atherosclerotic cardiovascular disease. Arch Med Res. 2015;46(5):328-38.

14. Bednarczyk M, Stege H, Grabbe S, Bros M. B2 Integrins-multi-functional leukocyte receptors in health and disease. Int J Mol Sci. 2020;21(4):1402.

15. Bindea G, Mlecnik B, Hackl H, Charoentong P, Tosolini M, Kirilovsky A, Galon J, et al. ClueGO: a cytoscape plug-in to decipher functionally grouped gene ontology and pathway annotation networks. Bioinformatics. 2009;25(8):1091-3.

16. Biros E, Karan M, Golledge J. Genetic variation and atherosclerosis. Curr Genomics. 2008;9(1):29-42.

17. Bonneau E, Neveu B, Kostantin E, Tsongalis G, De Guire V. How close are miRNAs from clinical practice? A perspective on the diagnostic and therapeutic market. Ejifcc. 2019;30(2):114.

18. Calway T, Kim GH. Harnessing the therapeutic potential of MicroRNAs for cardiovascular disease. J Cardiovasc Pharmacol Ther. 2015;20(2):131-43.

19. Chen L, Zhang Y-H, Zhang Z, Huang T, Cai Y-D. Inferring novel tumor suppressor genes with a protein-protein interaction network and network diffusion algorithms. Mol Therapy-Methods Clin Develop. 2018;10:57-67.

20. Conte F, Fiscon G, Licursi V, Bizzarri D, D’Antò T, Farina L, Paci P. A paradigm shift in medicine: a comprehensive review of network-based approaches. Biochimica et Biophysica Acta (BBA)-Gene Regulatory Mechanisms. 2020;1863(6):194416.

21. Crowther MA. Pathogenesis of atherosclerosis. ASH Education Program Book. 2005;2005(1):436-41.

22. Daugherty A, Manning MW, Cassis LA. Angiotensin II promotes atherosclerotic lesions and aneurysms in apolipoprotein E-deficient mice. J Clin Investig. 2000;105(11):1605-12.

23. Daugherty A, Rateri DL, Lu H, Inagami T, Cassis LA. Hypercholesterolemia stimulates angiotensin peptide synthesis and contributes to atherosclerosis through the AT1A receptor. Circulation. 2004;1 10(25):3849-57.

24. Dong $X$, Park S, Lin X, Copps $K$, Yi X, White MF. Irs 1 and Irs 2 signaling is essential for hepatic glucose homeostasis and systemic growth. J Clin Investig. 2006;116(1):101-14

25. Fagerholm SC, Guenther C, Llort Asens M, Savinko T, Uotila LM. Beta2Integrins and interacting proteins in leukocyte trafficking, immune suppression, and immunodeficiency disease. Front Immunol. 2019;10:254

26. Falcone R, Conte F, Fiscon G, Pecce V, Sponziello M, Durante C, Verrienti A, et al. BRAF V600E-mutant cancers display a variety of networks by SWIM analysis: Prediction of vemurafenib clinical response. Endocrine. 2019;64(2):406-13.

27. Finney AC, Stokes KY, Pattillo CB, Orr AW. Integrin signaling in atherosclerosis. Cell Mol Life Sci. 2017;74(12):2263-82.

28. Fiscon G, Conte F, Licursi V, Nasi S, Paci P. Computational identification of specific genes for glioblastoma stem-like cells identity. Sci Rep. 2018;8(1):1-10

29. Fountain JH, Lappin SL. Physiology, renin angiotensin system. (2017)
30. Grimaldi AM, Conte F, Pane K, Fiscon G, Mirabelli P, Baselice S, Salvatore $M$, et al. The new paradigm of network medicine to analyze breast cancer phenotypes. Int J Mol Sci. 2020;21(18):6690.

31. Hanna J, Hossain GS, Kocerha J. The potential for microRNA therapeutics and clinical research. Front Genet. 2019:10:478.

32. https://www.world-heart-federation.org/world-heart-day/world-heartday-2019/cvds

33. https://go.drugbank.com/

34. https://www.ncbi.nlm.nih.gov/gene

35. https://string-db.org/

36. Karnik SS, Unal H, Kemp JR, Tirupula KC, Eguchi S, Vanderheyden PM, Thomas WG. Angiotensin receptors: interpreters of pathophysiological angiotensinergic stimulis. Pharmacol Rev. 2015;67(4):754-819.

37. Keidar S. Angiotensin, LDL peroxidation and atherosclerosis. Life Sci. 1998;63(1):1-11.

38. Kota RS, Ramana CV, Tenorio FA, Enelow RI, Rutledge JC. Differential effects of lipoprotein lipase on tumor necrosis factor-a and interferonY-mediated gene expression in human endothelial cells. J Biol Chem. 2005;280(35):31076-84.

39. Li J, Chen L, Wang S, Zhang Y, Kong X, Huang T, Cai Y-D. A computational method using the random walk with restart algorithm for identifying novel epigenetic factors. Mol Genet Genomics. 2018;293(1):293-301.

40. Li L, Wang Y, An L, Kong X, Huang T. A network-based method using a random walk with restart algorithm and screening tests to identify novel genes associated with Menière's disease. PLoS ONE. 2017;12(8):e0182592.

41. Licursi V, Conte F, Fiscon G, Paci P. MIENTURNET: an interactive web tool for microRNA-target enrichment and network-based analysis. BMC Bioinformatics. 2019;20(1):1-10.

42. Lu H, Balakrishnan A, Howatt DA, Wu C, Charnigo R, Liau G, Daugherty $A$, et al. Comparative effects of different modes of renin angiotensin system inhibition on hypercholesterolaemia-induced atherosclerosis. Br J Pharmacol. 2012;165(6):2000-8.

43. Lu H, Wu C, Howatt DA, Balakrishnan A, Moorleghen JJ, Chen X, Crooke RM, et al. Angiotensinogen exerts effects independent of angiotensin II. Arterioscler Thromb Vasc Biol. 2016;36(2):256-65.

44. Luo X, Pan Z, Shan H, Xiao J, Sun X, Wang N, Qi X-Y, et al. MicroRNA-26 governs profibrillatory inward-rectifier potassium current changes in atrial fibrillation. J Clin Investig. 2013;123(5):1939-51.

45. Lusis AJ, Mar R, Pajukanta P. Genetics of atherosclerosis. Annu Rev Genomics Hum Genet. 2004;5:189-218.

46. Ma K, Miao Y, Gao Y, Tian J, Gao L, Ye D, Qin X. Increasing the level of IRS-1 and insulin pathway sensitivity by natural product Carainterol A. Molecules. 2016;21(10):1303.

47. Mardilovich K, Pankratz SL, Shaw LM. Expression and function of the insulin receptor substrate proteins in cancer. Cell Commun Signal. 2009;7(1):1-15.

48. Marques LR, Diniz TA, Antunes BM, Rossi FE, Caperuto EC, Lira FS, Gonçalves DC. Reverse cholesterol transport: molecular mechanisms and the non-medical approach to enhance HDL cholesterol. Front Physiol. 2018;9:526

49. Matsumoto M, Ogawa W, Teshigawara K, Inoue H, Miyake K, Sakaue H, Kasuga M. Role of the insulin receptor substrate 1 and phosphatidylinositol 3-kinase signaling pathway in insulin-induced expression of sterol regulatory element binding protein $1 \mathrm{c}$ and glucokinase genes in rat hepatocytes. Diabetes. 2002;51(6):1672-80.

50. Mead JR, Irvine SA, Ramji DP. Lipoprotein lipase: structure, function, regulation, and role in disease. J Mol Med. 2002;80(12):753-69.

51. Mead JR, Ramji DP. The pivotal role of lipoprotein lipase in atherosclerosis. Cardiovasc Res. 2002;55(2):261-9.

52. Merched A, Tollefson K, Chan L. B2 integrins modulate the initiation and progression of atherosclerosis in low-density lipoprotein receptor knockout mice. Cardiovasc Res. 2010;85(4):853-63.

53. Monchusi B, Kaur M. microRNAs targeting cellular cholesterol: implications for combating anticancer drug resistance. Genes Cancer. 2020;11(1-2):20.

54. Olivecrona G. Role of lipoprotein lipase in lipid metabolism. Curr Opin Lipidol. 2016;27(3):233-41.

55. Paci P, Fiscon G, Conte F, Licursi V, Morrow J, Hersh C, Silverman EK, et al. Integrated transcriptomic correlation network analysis identifies COPD molecular determinants. Sci Rep. 2020;10(1):1-18. 
56. Paci P, Fiscon G, Conte F, Wang R-S, Farina L, Loscalzo J. Gene co-expression in the interactome: moving from correlation toward causation via an integrated approach to disease module discovery. NPJ Syst Biol Appl. 2021;7(1):1-11.

57. Pan Y, Lu L, Chen J, Zhong Y, Dai Z. Identification of potential crucial genes and construction of microRNA-mRNA negative regulatory networks in osteosarcoma. Hereditas. 2018;155(1):1-8.

58. Panebianco V, Pecoraro M, Fiscon G, Paci P, Farina L, Catalano C. Prostate cancer screening research can benefit from network medicine: an emerging awareness. NPJ Syst Biol Appl. 2020;6(1):1-6.

59. Pirahanchi Y, Sharma S. Biochemistry, lipoprotein lipase. StatPearls [Internet]. (2020)

60. Previs SF, Withers DJ, Ren J-M, White MF, Shulman GI. Contrasting effects of IRS-1 versus IRS-2 gene disruption on carbohydrate and lipid metabolism in vivo. J Biol Chem. 2000;275(50):38990-4.

61. Qin L, Chen Y, Niu Y, Chen W, Wang Q, Xiao S, Zhao X, et al. A deep investigation into the adipogenesis mechanism: profile of microRNAs regulating adipogenesis by modulating the canonical Wnt/ $\beta$-catenin signaling pathway. BMC Genomics. 2010;11(1):1-10.

62. Romaine SP, Tomaszewski M, Condorelli G, Samani NJ. MicroRNAs in cardiovascular disease: an introduction for clinicians. Heart. 2015;101(12):921-8.

63. Ross $\mathrm{S}$, Gerstein H, Paré G. The genetic link between diabetes and atherosclerosis. Can J Cardiol. 2018;34(5):565-74.

64. Saito R, Smoot ME, Ono K, Ruscheinski J, Wang P-L, Lotia S, Ideker T, et al. A travel guide to Cytoscape plugins. Nat Methods. 2012;9(11):1069.

65. Sarajlić A, Janjić V, Stojković N, Radak D, Pržulj N. Network topology reveals key cardiovascular disease genes. PLoS ONE. 2013;8(8):e71537.

66. Singh KD, Karnik SS. (2016). Angiotensin receptors: structure, function, signaling and clinical applications. J Cell Signal 1(2).

67. Singh RB, Mengi SA, Xu Y-J, Arneja AS, Dhalla NS. Pathogenesis of atherosclerosis: A multifactorial process. Exp Clin Cardiol. 2002;7(1):40.

68. Song P, Fang Z, Wang H, Cai Y, Rahimi K, Zhu Y, Rudan I, et al. Global and regional prevalence, burden, and risk factors for carotid atherosclerosis: a systematic review, meta-analysis, and modelling study. Lancet Glob Health. 2020;8(5):e721-9.

69. Szklarczyk D, Gable AL, Nastou KC, Lyon D, Kirsch R, Pyysalo S, Bork P, et al. The STRING database in 2021: customizable protein-protein networks, and functional characterization of user-uploaded gene/measurement sets. Nucleic Acids Res. 2021;49(D1):D605-12.

70. Szklarczyk, D., Morris, J. H., Cook, H., Kuhn, M., Wyder, S., Simonovic, M., Bork, P., et al (2016). The STRING database in 2017: quality-controlled protein-protein association networks, made broadly accessible. Nucleic Acids Research, gkw937.

71. Takahashi M, Yagyu H, Tazoe F, Nagashima S, Ohshiro T, Okada K, Ishibashi $\mathrm{S}$, et al. Macrophage lipoprotein lipase modulates the development of atherosclerosis but not adiposity. J Lipid Res. 2013;54(4):1124-34.

72. Tieri, P., Farina, L., Petti, M., Astolfi, L., Paci, P., \& Castiglione, F. (2019). Network inference and reconstruction in bioinformatics.

73. Udhaya Kumar S, Thirumal Kumar D, Bithia R, Sankar S, Magesh R, Sidenna $\mathrm{M}$, Zayed $\mathrm{H}$, et al. Analysis of differentially expressed genes and molecular pathways in familial hypercholesterolemia involved in atherosclerosis: a systematic and bioinformatics approach. Front Genet. 2020;11:734.

74. Van Rooij E, Sutherland LB, Thatcher JE, DiMaio JM, Naseem RH, Marshall WS, Olson EN, et al. Dysregulation of microRNAs after myocardial infarction reveals a role of miR-29 in cardiac fibrosis. Proc Natl Acad Sci. 2008;105(35):13027-32.

75. Vickers KC, Shoucri BM, Levin MG, Wu H, Pearson DS, Osei-Hwedieh D, Sethupathy $P$, et al. MicroRNA-27b is a regulatory hub in lipid metabolism and is altered in dyslipidemia. Hepatology. 2013;57(2):533-42.

76. Walayat, A., Yang, M., \& Xiao, D. (2018). Therapeutic implication of miRNA in human disease Antisense therapy: IntechOpen.

77. Wang J, Song Y, Zhang Y, Xiao H, Sun Q, Hou N, Zhan D, et al. Cardiomyocyte overexpression of miR-27b induces cardiac hypertrophy and dysfunction in mice. Cell Res. 2012;22(3):516-27.

78. Wang Y, Nishina PM, Naggert JK. Degradation of IRS1 leads to impaired glucose uptake in adipose tissue of the type 2 diabetes mouse model TALLYHO/Jng. J Endocrinol. 2009;203(1):65.

79. Weinstock PH, Bisgaier CL, Aalto-Setälä K, Radner H, Ramakrishnan R, Levak-Frank S, Breslow JL, et al. Severe hypertriglyceridemia, reduced high density lipoprotein, and neonatal death in lipoprotein lipase knockout mice Mild hypertriglyceridemia with impaired very low density lipoprotein clearance in heterozygotes. J Clin Investig. 1995;96(6):2555-68.

80. Wu H, Gower RM, Wang H, Dai Perrard X-Y, Ma R, Bullard DC, Simon SI, et al. Functional role of $\mathrm{CD} 11 \mathrm{C}+$ monocytes in atherogenesis associated with hypercholesterolemia. Circulation. 2009;119(20):2708.

81. Wu P, Wang Q, Jiang C, Chen C, Liu Y, Chen Y, Zeng Y. MicroRNA-29a is involved lipid metabolism dysfunction and insulin resistance in C2C12 myotubes by targeting PPARS. Mol Med Rep. 2018;17(6):8493-501.

82. Xu X, Zhao Z, Li G. The therapeutic potential of MicroRNAs in Atrial Fibrillation. Mediators of inflammation, 2020.

83. Yoke Yin C, So Ha T, Abdul Kadir K. Effects of glycyrrhizic acid on peroxisome proliferator-activated receptor gamma (PPAR), lipoprotein lipase (LPL), serum lipid and HOMA-IR in rats. PPAR Research. (2010)

84. Yoon J, Blumer A, Lee K. An algorithm for modularity analysis of directed and weighted biological networks based on edge-betweenness centrality. Bioinformatics. 2006;22(24):3106-8.

85. Yosten GL, Samson WK. Cardiovascular neuroendocrinology. Handbook of Neuroendocrinology (pp 307-327): Elsevier. (2012)

86. Yu X-H, Zhang D-W, Zheng X-L, Tang C-K. Cholesterol transport system: an integrated cholesterol transport model involved in atherosclerosis. Prog Lipid Res. 2019;73:65-91.

87. Zaman MA, Oparil S, Calhoun DA. Drugs targeting the renin-angiotensin-aldosterone system. Nat Rev Drug Discovery. 2002;1 (8):621-36.

88. Zhang L, Li X, Tai J, Li W, Chen L. Predicting candidate genes based on combined network topological features: a case study in coronary artery disease. PLOS ONE. 2012;7(6):e39542.

89. Zhang $S$, Cheng Z, Wang Y, Han T. The risks of miRNA therapeutics: in a drug target perspective. Drug Des Dev Ther. 2021;15:721.

90. Zhang W, Wang Q, Feng Y, Chen X, Yang L, Xu M, Gao D, et al. MicroRNA26 a protects the heart against hypertension-induced myocardial fibrosis. J Am Heart Assoc. 2020;9(18):e017970.

91. Zhang Y, Sun X, Icli B, Feinberg MW. Emerging roles for microRNAs in diabetic microvascular disease: novel targets for therapy. Endocrinol Rev. 2017;38(2):145-68.

92. Zhang Y, Zeng T, Chen L, Ding S, Huang T, Cai Y-D. Identification of COVID19 infection-related human genes based on a random walk model in a virus-human protein interaction network. BioMed Res Int. 2020;2020:1.

93. Zhou S-S, Jin J-P, Wang J-Q, Zhang Z-G, Freedman JH, Zheng Y, Cai L. miRNAS in cardiovascular diseases: potential biomarkers, therapeutic targets and challenges. Acta Pharmacol Sin. 2018;39(7):1073-84.

\section{Publisher's Note}

Springer Nature remains neutral with regard to jurisdictional claims in published maps and institutional affiliations.

\footnotetext{
Ready to submit your research? Choose BMC and benefit from:

- fast, convenient online submission

- thorough peer review by experienced researchers in your field

- rapid publication on acceptance

- support for research data, including large and complex data types

- gold Open Access which fosters wider collaboration and increased citations

- maximum visibility for your research: over $100 \mathrm{M}$ website views per year
}

At BMC, research is always in progress.

Learn more biomedcentral.com/submissions 